\title{
Warmth in Groups and Satisfaction with Supervision
}

\author{
Kamarul Zaman Ahmad \\ Faculty of Business \& Accountancy \\ University of Malaya, Kuala Lumpur, Malaysia \\ E-mail: drkamphd@yahoo.com
}

Received: May 5, 2010 Accepted: July 16, $2010 \quad$ doi:10.5539/ijbm.v6n5p129

\begin{abstract}
Most previous studies of Person-Environment fit have failed to use objective measures of the work environment that are independent of the person examined. This study aims to further develop and improve the operationalisation of Person-Environment fit by investigating objective fit and the interaction effect of group size on the relationship between the personality trait of warmth and satisfaction with supervision. Group size (obtained from company records) was the objective measure of the work environment and warmth was measured by the 16PF (Cattel et al 1987). On the basis of data from 257 shop-floor workers in a factory in Wales, UK, results of hierarchical multiple regression generally indicated that the relationship between warmth and satisfaction with supervision are moderated by group size such that the relationship is positive in small groups and negative in large groups.
\end{abstract}

Keywords: P-E fit, objective fit, Warmth, Group size, Supervision satisfaction, Hierarchical multiple regression

\section{Introduction}

Parsons' (1909) seminal work appears to be the first to have stressed the importance of both person and environment variables in vocational choice. There is an abundance of research that examined the degree of fit between the person and the environment i.e., person-environment fit or P-E fit and how that is associated with satisfaction. Kristof, Zimmerman and Johnson (2005) have given one of the most up-to-date and comprehensive review of the previous studies on P-E fit. So have Piasentin and Chapman (2006) and Verquer, Beehr and Wagner (2003) when they conducted a meta-analytic review of 46 and 21 studies of P-E fit respectively. Yet, despite the illuminating articles by Kristof et al., (2005, 1996), other researchers (Wessel, Ryan and Oswald, 2008) appear somewhat confused especially when it comes to operationalising objective fit. True objective fit, is the fit when the measures of the environment are taken from a separate source and not from the respondent who is providing the information relating to the 'person' variable (Kristof et al., 2005, 1996). One example of a measure of the environment that can be obtained from company records is group size. Until today, there has not been a single P-E fit study that addressed this issue specifically. Moreover, most P-E fit research looked at various conceptualisations of personality or values when measuring the person. However, warmth, as a measure of the person has previously been ignored in P-E fit literature.

The objective of this study is to examine the degree of objective fit between the person and the environment and how that is associated with satisfaction. More precisely, it examines the interaction between warmth (person) and group size (an objective measure of the work environment) and how that is associated with satisfaction with supervision (dependent variable). For instance, does the relationship between warmth and satisfaction with supervision vary with group sizes? This is the main research question that this study aims to answer.

\section{Literature Review}

\subsection{Person-Environment fit or P-E fit}

P-E fit was summarised by Edwards (1996: 292) as follows: "In essence, P-E fit embodies the premise that attitudes, behaviour and other individual level outcomes result not from the person or environment separately, but rather from the relationship between the two (Lewin, 1951; Murray, 1938; Pervin, 1989)." Kristof $(2005,1996)$, Piasentin and Chapman (2006) and Verquer, Beehr and Wagner (2003) have done a thorough literature review and as such, the bulk of it will not be reproduced here. Rather, what is discussed in this paper is the two main ways of conceptualising fit - direct and indirect. This eventually leads to the researcher's justification and recommendation that the environment should be measured objectively (in order to get a correspondingly objective measure of fit). 
Kristof et al., $(2005,1996)$ indicated that some researchers have used direct measures of fit i.e., by asking people explicitly whether they believe a good fit exists. Posner, Kouzes and Schmidt (1985) used such a method. In their study, managers directly rated how compatible their values were with those of their organizations and how often they had to compromise personal principles to meet organizational expectations. This method is plagued by the consistency bias (i.e., "I think that I fit well, so I must be satisfied with my job") and is therefore not adopted in the current research. Due to this drawback, some researchers have relied on indirect measures to assess fit. According to Kristof (1996), indirect measures are more reliable because the respondent is asked to rate the individual separately from the environment without being asked to assess the degree of fit. This method (hereinafter called "the moderator approach") does not insist on commensurate measures. The person and the environment can be measured separately (as they should be), using entirely different instruments. The nature and ranges of the two scales can be entirely different. This does not require the respondent to assess fit either directly or indirectly. In fact, it makes it virtually impossible for the respondent to even attempt to assess fit. In this respect, the moderator approach is superior in that the consistency bias inherent in the direct measurement of fit approach can be totally eliminated.

Furthermore, it can be argued that the biggest advantage with this method is that objective measures of the environment can be used. Objective measures are measures that do not require any conceptual transformation on the part of the respondent. Hence, it is entirely independent of the person (respondent). Unfortunately, many studies that have used the moderator approach have failed to capitalise on this advantage. For example, in Lee, Ashford and Bobko (1990), control was measured perceptually by the respondent. In Barrick and Mount (1993), autonomy was measured perceptually. Edwards (1991) criticised direct measures primarily because they confound the constructs of the person and environment, thereby preventing the estimation of their independent effects. Yet, amazingly, he did just that (in Edwards, 1996) - environmental supplies and personal values (S-V) fit were measured by asking respondents how much of each task was involved in their job and how much of each task they preferred! This procedure was also employed in another research by Livingstone, Nelson and Barr (1997) who also conducted a study that employed a similar measure of S-V fit in their research.

Although Edwards (1991) pointed out that there is a real need for future research to use objective measures (of either the person or the environment), he has not done so himself and there appears to be none done by others reported even until today. Thus, there is a pressing need for a research to be conducted that uses a truly objective measure i.e., one which is obtained from a source other than the respondent.

\subsection{Group Size}

Group size, a measure that can be obtained from company records, is one aspect of the work environment that can be measured objectively. Social interactions in groups are frequently characterized by conflicts between personal and collective interests (De Cremer and Leonardelli, 2003). Thus, it is not surprising that research has shown that smaller groups establish and maintain higher levels of communication (Lowry, Roberts, Romano, Cheney and Hightower, 2006) whereas larger groups have reported lower satisfaction (Frank and Anderson, 1971; Shaw, 1981; Slater, 1958; Worthy, 1950). As group size increases, almost every group experiences some degradation in group communication process due to social loafing (Chidambaram and Tung, 2005; Liden, Wayne, Jaworski, and Bennett, 2009). Dissatisfaction with large groups is also reflected by greater absenteeism and personnel turnover (Shaw, 1981). However, small groups are not always better than larger groups. Aiken and Wong (2003) discovered that for idea generation, groups may not be effective until they reach a certain size. However, this apparent controversy over which size is better can be summed up by Worthy (1950) who stated that mere size is unquestionably one of the most important factors in determining the satisfaction of employees and dissatisfaction can have serious consequences for the company.

\subsection{The personality trait of warmth}

According to Cattell, Eber and Tatsuoka (1992), people whose traits are high in warmth value personal relationships whereas those whose traits are low in warmth are more impersonal. People whose traits are high in warmth are interested in people, easily affected and more generous in personal relationships. On the other hand, people whose traits are low in warmth are awkwardly aloof and prefer to work alone. They prefer things like logic and machinery to people. From the descriptions above, it would be reasonable to say that people whose traits are high in warmth place a higher importance on the relationship between them and their supervisor than those low in warmth who are not interested in relationships and prefer to be left alone to do their work. Consider the following situation: Supervisors hold meetings with the groups about once a month, or more frequently, when there are problems or new procedures to discuss. These meetings are held between the supervisor and everyone belonging to the group. When supervisors meet with subordinates collectively in a group, supervisors are able to give more 
personal attention to subordinates if they belong to small groups rather than large groups. This is because supervisors find it difficult to give everyone in the large group a high degree of attention where there are so many of them. This can be contrasted to small groups where each subordinate has a much better chance of having their voices heard during group meetings with their supervisor. The theory accordingly is that respondents whose traits are high in warmth will value more personal attention from their supervisors and will consequently be more satisfied with supervision if they work in small groups. This theory leads to the following hypothesis:

H1: The association between warmth scores and satisfaction with supervision scores will be moderated by group size such that the association will be negative for large groups and positive for small groups.

\section{Research Methodology}

\subsection{Sample}

Data on two-hundred and fifty-seven shop-floor workers who worked in groups were collected from a light manufacturing assembly plant in Wales, UK. The entire population of the workers on the shop-floor was three hundred. So, this research is almost a census for the organization. One person refused to participate in the survey and two other resigned before the test could be completed. The majority of the sample was females - there were one hundred and eighty females $(70 \%)$ and seventy-seven males (30\%). The average age of the sample was thirty-five (see Table 1). Test administration took place over a period of three months in the factory. The collection of data on the employees in the sample took place in sessions at which, from three to fifteen employees were present. Subjects were asked to supply their names to identify their work groups. Every attempt was made to reduce the respondents' worries and anxieties about participating in the research and that this research was mainly for academic purposes. As this study was conducted in the UK no prior ethics approval was required. Nevertheless, anonymity of the respondents were preserved as promised and the rights of the individual respondents were not infringed.

The rationale for choosing to study only one organization was that most P-E fit studies examine differences between rather than within jobs and professions despite the fact that there may be very large differences between them (Furnham, Toop Lewis and Fisher, 1995). In the said article, one of the three reported studies explored P-E fit among two groups of specialist nurses (paediatrics and intensive therapy) (twenty-six and twenty-nine respectively) and one group of general medical nurses (twenty-five). Another reason why data was collected from only one organization was to reduce the variance due to factors which are not measured but must still be controlled (or at least be subjected to some attempt at control). Examples of such factors are differences in company policies, company size, industry sector and geographical location. Adkins Ravlin and Meglino (1996), in their research (which has some similarities with this research), also confined their study to one organization (one-hundred and ninety-one production workers). Thus, it will actually be counter-productive to collect the data from different organizations in this case. Bouchard (1983: 367) stated "Many field studies involve only one organization or subject. While the one case study is generally sterile scientifically.... it need not be. ... A well chosen single case may seriously threaten a traditionally accepted hypothesis."

\subsection{Measures}

Group size was measured by the number of workers in the work team as shown in the company records. Warmth was measured by 6 items in the 16 PF (Cattell et al., 1992) that measures Factor I. One such example is "I think what people say in poetry could be put exactly in plain prose." Scoring instructions are provided by Cattell et al., (1992). The $16 \mathrm{PF}$ remains a popular questionnaire until today and still being used by many researchers (Forero, Pujol, Olivares and Pueyo, 2009; Wang and Xu 2008; Cousineau, Hall Mel, Rosik and Hall, 2007). Supervision satisfaction was measured by numerical scales adapted from another P-E fit study (Meir, Hadas and Noyfeld, 1997), ranged from 1 to 20 (1 being lowest and 20 being the highest level of satisfaction).

\subsection{Procedure for defining small and large groups}

The procedure for defining group size is as follows: The entire sample was split into three equal groups according to group size i.e., small, medium and large. Subsequently, only the large groups were compared with the small groups, the two being at the extremes. The basis of dividing the groups as such was to adopt a compromise between two opposing recommendations. Roberts and Foti (1998) and Adkins, Ravlin and Meglino (1996) used median splits to divide their sample into two equal groups and then proceeded to compare one group with another. This method has the advantage of utilising the entire sample in the analyses. However, the disadvantage with this method is that it will inevitably include those respondents who are at the borderline i.e., just below or just above the mean of the variable under investigation. As such, it was felt that three groups rather than two should be formed 
and the analyses restricted to the comparison of the two extreme groups only. For instance, Barrick and Mount (1993) divided their entire sample according to $+1,0$ and -1 standard deviation. However, the outcome was that there were rather small sub-samples at both ends of the spectrum $(\mathrm{n}=59$ at the +1 standard deviation above the mean and $n=49$ at the -1 standard deviation below the mean) with a high 'wastage' $(n=149)$ in the middle of the range. The end result was a compromise and the entire sample was divided into three equal groups. The SPSS commands of "summarise - frequencies - statistics - cut points for 3 equal groups" were used to determine the appropriate maximum values for small groups and minimum values for large groups. The result was that groups comprising between two and seven members are classified as small, between eight to fourteen are considered medium, and between fifteen and twenty are considered large.

\section{Results}

\subsection{Reliability}

Cronbach coefficient alpha reliability scores for warmth were 0.73 . Cronbach coefficient alpha reliability scores for supervision satisfaction are unavailable since they are one-item measures. Descriptives statistics are given in Table 1. Correlation coefficients between the main variables are given in Table 2. Generally, warmth is not significantly correlated with group size and supervision satisfaction.

\section{Insert Table 1 here}

Insert Table 2 here

\subsection{Hierarchical Regression}

Hierarchical multiple regression were conducted to test the significance of the moderating effect of group size. More specifically, tests of hierarchical regression were used to determine the significance of the interaction term (warmth multiplied by the group size) on supervision satisfaction. In its simplest form, a hierarchical (sometimes called sequential) analysis involves entering the IVs singly or cumulatively in a prespecified sequence. The R2 and partial coefficients are examined at each step. In hierarchical regression "the researcher determines the order in which IVs are entered into the equation," taking into account logical or theoretical considerations (Tabachnick and Fidell, 1996: 149). The IVs ( $u$ and v) can be entered in block 1 of the analyses using the command "Enter" in the SPSS program. The interaction term $(\mathrm{u} \times \mathrm{v})$ can then be introduced into the equation in block two. The term "introduced" rather than "entered" is used because, in block two, the interaction term is accepted into the equation only if, together with the individual component variables, it explains a variance in the dependent variable significantly more $(\mathrm{p}<.05)$ than the individual component variables alone. If the interaction term can significantly $(\mathrm{p}<.05)$ improve the predictive power of the equation, then it will form part of the regression equation. If it fails to significantly improve the R2 (squared semi-partial correlation), then the SPSS programme will not include the interaction term from the regression equation. The command used in block 2 was "Stepwise". Results of hierarchical multiple regression analyses are given in Table 3.

During the hierarchical multiple regression tests, the interaction term (warmth multiplied by the group size) was successfully accepted into the regression equation after the individual components (warmth and group size) have been entered into the equation. The results showed suppport for H1A in so far as group size was a significant moderator of the warmth-satisfaction relationships. Full details of the values of $\mathrm{R}^{2}$ were set out in Table 3 . For instance Table 3, Column 3 showed the $\mathrm{R}^{2}$ values when only the warmth variable and the group size were entered into the regression equation. Column 4 of the same table show the $\mathrm{R}^{2}$ values when the warmth variable and group size as well as the interaction term were all in the equation. Column 5 of the same table show the change in $\mathrm{R}^{2}$ as a result of introducing the interaction term.

\section{Insert Table 3 here \\ Insert Table 4 here}

Table 4 shows the values of $b$ and the correlation coefficients in three scenarios: all cases, large groups only and small groups only. Figures 1 shows the graphs for large groups, small groups and all cases, for supervision satisfaction.

\section{Insert Figure 1 here}

Thus, the results of this research showed support for the H1A in that the relationship between warmth and supervision satisfaction, were moderated by group size in so far as the said relationships were negative in large groups and positive in small groups, as indicated by the values of $b$ and as evident from Figure 1. However, only the correlation between warmth and supervision satisfaction was significantly positive in small groups (.199, $\mathrm{p}$ $<.01)$. Correlations between warmth and supervision satisfaction in large groups were not significant. 


\section{Conclusion}

The results of this study lends support to the P-E fit theory in that people who fit in their environment tend to be more satisfied than those who do not. People whose traits are high in warmth fit in working environments where supervision is more personalised and individualised. As mentioned earlier, when supervisors meet with subordinates collectively in a group, supervisors are able to give more personal attention to subordinates if they belong to small groups rather than large groups. Supervisors may find it difficult to give everyone in the large group a high degree of attention where there are so many of them. In small groups, each subordinate has a much better chance of having a higher degree of interaction during group meetings with their supervisor. Results seem to suggest that, people whose traits are high in warmth have a tendency to be less satisfied with their supervisors if they are made to work in large groups. People whose traits are high in warmth are more satisfied with their supervisors if they work in small groups where the supervisors are able to give them more personal attention.

From the managerial perspective, results seem to suggest that people whose traits are high in warmth should be made to work in smaller groups, where they will be more satisfied with their supervisors and not in larger groups where they will be less satisfied. However, to be able to do so, management would have to find out which workers have traits high in warmth and which have traits low in warmth. This would necessitate personality tests to be conducted on all the workers, a decision which may or may not be popular with the management or the workers. From the person-environment perspective, managers have two choices. Change the person or change the environment. Managers could either select only those people whose traits are low in warmth, or they could limit the group sizes so as not to exceed ten in number. The second alternative seems simpler, and this action would probably reduce the occurrence of social loafing hence resulting in increased productivity of the average worker. In addition, management can make individual contributions more visible.

\section{Limitations}

Since this study is cross sectional, a conclusion cannot be drawn about the direction of causality in these findings. However, it is reasonable to hold that the interaction between warmth and group size was for the most part responsible for changes in satisfaction rather than the reverse as the view taken in this research is that personality traits are relatively stable over long periods of time. Another weakness is that the moderator analyses were conducted in a single company. However, this can be argued to be a strength as many variables such as, company policies, company size, industry sector and geographical location are controlled because the study was conducted in the same factory. Such variables, if uncontrolled, may have varying effects on the moderator, the independent and dependent variables. The findings of this study can easily benefit from replication studies conducted in other companies with the same characteristics. It is also important to stress here that group size per se may not moderate the relationships found in this study. For instance, if supervisors are not interested in maintaining close relationships with their subordinates there may be no moderating effect of group size on the warmth-supervision satisfaction relationship. It is important that future replication studies fully replicate all the different characteristics of large and small groups and of the company itself. In defence, it can be argued that the differences that exist between small and large groups as well as the characteristics of the company are by no means unique and it is possible to find the same in other companies. Finally, lateral relationships in medium sized groups were not examined and future research should be carried out to investigate this.

\section{References}

Adkins, C. L., Ravlin, E. C., \& Meglino, B. (1996). Value congruence between co-workers and it's relationship to work outcomes. Group and Organisation Management, 21(4), 439-460.

Aiken, M., \& Wong, Z. (2003). The effect of group size on electronic idea generation. International Journal of Human Resources Development and Management, 3(3), 265.

Barrick, M. R., \& Mount, M. K. (1993). Autonomy as a moderator of the relationships between the Big Five personality dimensions and job performance. Journal of Applied Psychology, 78(1), 111-118.

Bouchard T. J. JR. (1983). Field research methods: interviewing, questionnaires, participant observation, systematic observation, unobtrusive measures. In Dunnette, M. D. (Ed.), Handbook of Industrial and Organizational Psychology, John Wiley \& Sons.

Cattell, R. B., Eber, H. W., \& Tatsuoka, M. M. (1992). Handbook for the Sixteen Personality Questionnaire. Champaign Illinois: IPAT.

Chidambaram, L., \& Tung, L.L. (2005). Is out of sight, out of mind? An empirical study of social loafing in technology-supported groups. Information Systems Research, 16(2), 149-160. 
Cousineau, A. E., Hall Mel, E. L., Rosik, C. H., \& Hall, T. W. (2007). The 16 PF and marital satisfaction inventory as predictors of missionary job success. Journal of Psychology and Theology, 35(4), 317-327.

De Cremer, D., \& Leonardelli, G. J. (2003). Cooperation in social dilemmas and the need to belong: The moderating effect of group size. Group Dynamics: Theory, Research, and Practice, 7(2), 168-174.

Edwards, J. R. (1991). Person-job fit: a conceptual integration, literature review and methodological critique. International Review of Industrial/ Organizational Psychology, 6, 283-357.

Edwards, J. R. (1996). An examination of competing versions of the person-environment fit approach to stress. Academy of Management Journal, 39(2), 292-339.

Forero, C. G., Pujol, D. G., Olivares, A. M., \& Pueyo, A. A. (2009). A longitudinal model for predicting performance of police officers using personality and behavioural data. Criminal Justice and Behavior, 36(6), 591-606.

Frank, F., \& Anderson, L. R. (1971). Effects of task and group size upon group productivity and member satisfaction. Sociometry, 34, 135-149.

Furnham, A., Toop, A., Lewis, C., \& Fisher, A. (1995). P-E fit and job satisfaction: a failure to support Holland's theory in three British samples. Personality and Individual Differences, 19, 677-690.

Kristof, A. (1996). Person-organisation fit: an integrative review of it's conceptualisations, measurement, and implications. Personnel Psychology, 49, 1-49.

Kristof, A., Zimmerman, R. D., \& Johnson, E. C. (2005). Consequences of individuals' fit at work: a meta-analysis of person-job, person-organization, person-group and person-supervisor fit. Personnel Psychology, $58,281-342$.

Lee, C., Ashford, S. J., \& Bobko, P. (1990). Interactive effects of "Type A" behavior and perceived control on worker performance, job satisfaction and somatic complaints. Academy of Management Journal, 33(4), 870-881.

Lewin, K. (1951). Field Theory in Social Science. New York: Harper.

Liden, R., Wayne, S.J., Jaworski, R.A., \& Bennett, N. (2004). Social loafing: a field investigation. Journal of Management, 30(2), 285.

Livingstone, L. P., Nelson, D. L., \& Barr, S. H. (1997). Person-environment fit and creativity: an examination of supply-value and demand-ability versions of fit. Journal of Management, 23(2), $119-146$.

Lowry, P. B., Roberts, T. L., Romano N. C., Cheney, P. D., \& Hightower, R. T. (2006). The impact of group size and social presence on small group communication - does computer-mediated communication make a difference? Small Group Research, 37(6), 631-661.

Meir, E. I., Hadas, C., \& Noyfeld, M. (1997). Person-environment fit in small army units. Journal of Career Assessment, 5, 21-29.

Murray, H. A. (1938). Explorations in Personality. Boston: Houghton Mifflin.

Parsons, F. (1909). Choosing a Vocation. Boston, MA: Houghton Mifflin.

Pervin, L. A. (1989). Persons, situations, interactions: the history and a discussion of theoretical models. Academy of Management Review, 14(3), 350-360.

Piasentin, K. A., \& Chapman, D. S. (2006). Subjective person-organisation fit: Bridging the gap between conceptualisation and measurement. Journal of Vocational Behaviour, 69, 202-221.

Posner, B. Z., Kouzes, J. M., \& Schmidt, W. H. (1985). Shared values make a difference - an empirical test of corporate culture. Human Resources Management, 24(3), 293-309.

Roberts, H. E., \& Foti, R. J. (1998). Evaluating the interaction between self-leadership and work structure in predicting job satisfaction. Journal of Business and Psychology, 12(3), 257-267.

Shaw, M. E. (1981). Group Dynamics. The Psychology of Small Group Behavior. New York: McGraw Hill.

Slater, P. E. (1958). Contrasting correlates of group size. Sociometry, 21, 129-139.

Tabachnick, B. G. \& Fidell, L. S. (1996). Using Multivariate Statistics. 3rd edn. New York: Harpur Collins College Publishers.

Verquer, M. L., Beehr, T. A., \& Wagner, S. H. (2003). A meta-analysis of relations between person-organization fit and work attitudes. Journal of Vocational Behaviour, 63, 473-489. 
Wang, A. P., \& Xu, Y. (2008). Applicability of five-kind of personality inventory for college students' personality measurement: Comparison study with 16PF. International Journal of Psychology, 43(3), 480-480

Wessel, J. L., Ryan, A. M., \& Oswald, F. L. (2008). The relationship between objective and perceived fit with academic major, adaptability, and major-related outcomes. Journal of Vocational Behaviour, 72, 363-376.

Worthy, J. C. (1950). Organizational structure and employee morale. American Sociological Review, 15, $169-179$.

Table 1. Descriptives

\begin{tabular}{|l|l|l|}
\hline & Means & Standard deviation \\
\hline Warmth & 7.31 & 2.23 \\
\hline Satisfaction with supervision & 31.09 & 13.56 \\
\hline Age & 35.57 & 10.66 \\
\hline
\end{tabular}

$\mathrm{N}=257$

Table 2. Table of correlation coefficients

\begin{tabular}{|l|c|c|c|}
\hline & Warmth & Group Size & Satisfaction with Supervision \\
\hline Warmth & 1 & .779 & .364 \\
\hline Group size & .779 & 1 & $.171^{* *}$ \\
\hline Satisfaction with supervision & .364 & $-.171^{* *}$ & 1 \\
\hline
\end{tabular}

$* * \mathrm{p}<.01, * \mathrm{p}<.05, \mathrm{~N}=257$

Above diagonal $=$ significance

Below diagonal $=$ Pearson correlation coefficients

Table 3. Warmth and supervision satisfaction moderated by group size

\begin{tabular}{|c|c|c|c|c|}
\hline \multirow[t]{2}{*}{ Person Variable } & \multirow{2}{*}{$\begin{array}{l}\text { Supervision } \\
\text { Satisfaction }\end{array}$} & \multicolumn{3}{|c|}{ Hierarchical Multiple Regression } \\
\hline & & $\begin{array}{l}\mathrm{R}^{2} \text { with } \mathrm{P}, \mathrm{E} \text { but excluding } \\
\text { interaction term }\end{array}$ & $\begin{array}{l}\mathrm{R}^{2} \text { including } \\
\text { interaction term }\end{array}$ & $\Delta \mathrm{R}^{2}$ \\
\hline Warmth & H1 & .047 & .065 & .018 \\
\hline
\end{tabular}

Table 4. Values of $\mathrm{b}$ and the correlation coefficients

\begin{tabular}{|l|l|l|l|l|}
\hline HYPOTHESIS & $\begin{array}{l}\text { STATISTICAL } \\
\text { ANALYSIS }\end{array}$ & $\begin{array}{l}\text { ALL CASES } \\
(\mathrm{N}=257)\end{array}$ & $\begin{array}{l}\text { LARGE } \\
\text { GROUPS } \\
(\mathrm{N}=90)\end{array}$ & $\begin{array}{l}\text { SMALL } \\
\text { GROUPS } \\
(\mathrm{N}=97)\end{array}$ \\
\hline \begin{tabular}{l} 
H1 $\begin{array}{l}\text { Warmth \& satisfaction } \\
\text { with supervision }\end{array}$ \\
\cline { 2 - 5 }
\end{tabular} & Values of $\mathrm{b}$ & .0001 & -.1219 & .0685 \\
\cline { 2 - 5 } & Correlation coefficient & .070 & -.061 & $.199^{* *}$ \\
\hline
\end{tabular}

$* * \mathrm{p}<.01, * \mathrm{p}<.05, \mathrm{~N}=257$ 


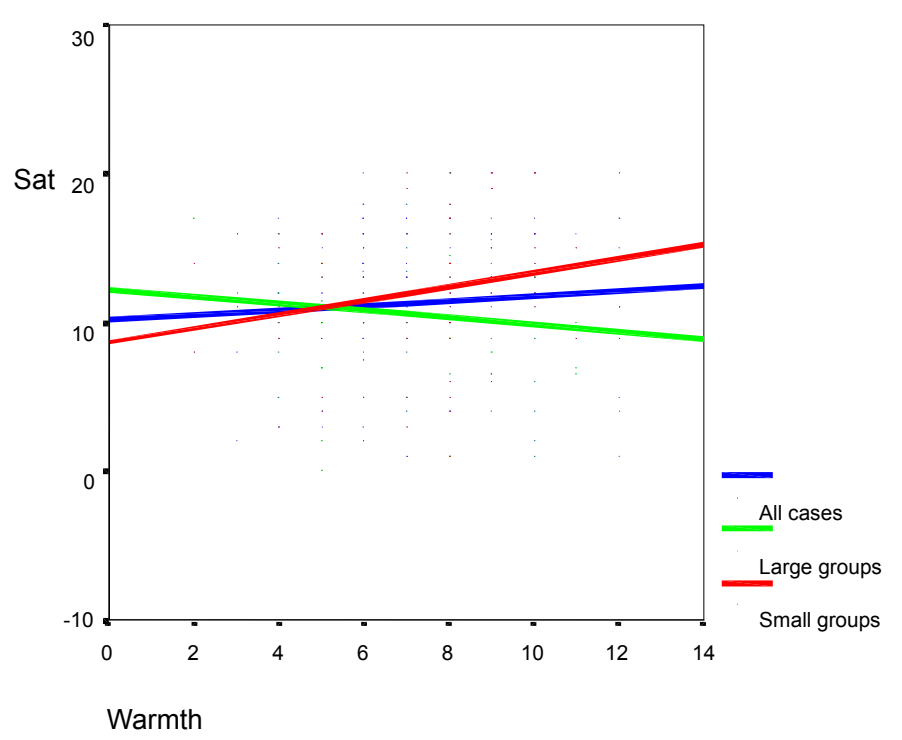

Figure 1. Interactional Effects of Group Size on the relationhip between Warmth and Satisfaction with Supervision 